\title{
Overshoot of curvature in visual apparent motion
}

\author{
DAVID H. FOSTER and SALVATORE GRAVANO \\ University of Keele, Keele, Staffordshire, England
}

\begin{abstract}
If a curved line and a straight line are presented briefly, one above the other, in sequence to the eye, then, under appropriate conditions, visual apparent motion is obtained. Subjects report that the illusory figure moving and changing from the curved line to the straight line appears to overshoot the latter, gaining a small curvature in the opposite sense. Three experiments are described. In the first, the magnitude of this apparent curvature was quantified as a function of the delay between the onsets of the curved line and straight line (the stimulus onset asynchrony, SOA). It is shown that overshoot in curvature cannot be attributed to inappropriate patterns of eye fixations. In the second experiment, the stimulus configuration was modified to reveal the contribution to apparent curvature of classical curvature-contrast effects. Curvature overshoot due to apparent motion alone was thus estimated as a function of SOA. In the third experiment, an analogous position overshoot was measured for apparent motion elicited by two brief sequentially presented parallel line segments. It is argued that a combination of such position overshoots cannot explain curvature overshoot. Two schemes of a more general kind that might be used to interpret curvature overshoot are then outlined. One scheme is based on a neural-net model of apparent motion, and the other on a functional model of apparent motion that operates by laws analogous to those governing real physical motion.
\end{abstract}

Visual apparent motion occurs when two suitably shaped and suitably timed, spatially resolvable stimuli are presented in sequence to the eye (Exner, 1875; Wertheimer, 1912). If the percept induced by these stimuli is of an object moving smoothly and continuously from one location to another, the phenomenon is sometimes called beta motion (Wertheimer, 1912; Kenkel, 1913; Kolers, 1972).

In the course of exploring beta motion elicited by the alternation of a briefly presented square and a briefly presented overlapping circle (as described by Kolers \& Pomerantz, 1971), we observed that, when the circle preceded the square, the illusory movement was not of a circle changing into a square, but rather of a circle changing into a concave pincushion figure. The effect is as if the illusory figure "overshoots" the form defined by the second "target" figure.

Overshoot of perceived position or distance in apparent motion, occurring, for example, with the illusory figure induced by the sequential flashing of two points or two parallel lines, has been reported pre-

We wish to thank S. R. Pratt and J. Thorson for indispensable advice and for critical readings of an earlier version of this report, D. M. MacKay, R. J. Mason, T. Morotomi, G. F. Pick, and R. S. Snelgar for much valuable discussion, and R. Knapper for technical assistance. S.G. was supported by an award from the Science Research Council. Some of the data reported here were contained in a communication read at the Oxford meeting of the Experimental Psychology Society, July 1981. The authors' complete address is: Department of Communication and Neuroscience, University of Keele, Keele, Staffordshire ST5 5BG, England. viously. Thus, under some conditions, Scholz (1924) obtained distance overshoots of $17 \%-44 \%$ of the interstimulus distance, and Neuhaus (1930) obtained overshoots of 9\%-34\%. Kolers and Touchstone (Note 1) observed smaller distance overshoots of $4 \%-8 \%$, but attributed these variations in apparent distance to involuntary eye movements occurring between the extinction of the first stimulus and the onset of the second.

The overshoot phenomenon described above, involving the sequential presentation of a circle and a square, may also be induced by sequentially presenting a straight line and a curved line, aligned as if they were the spatially overlapping parts of the original figures. For this reduced stimulus configuration, the phenomenon might be interpreted, by analogy with an overshoot in position, as an overshoot in curvature. The purpose of this study was to explore some of the possible processes that might give rise to curvature overshoot. Although we consider effects of stimulus time course and geometry, our approach is not intended to be explicitly parametric.

The organization of the study is as follows. Experiment 1 measures the magnitude of apparent curvature of a briefly presented straight line as a function of the delay between its onset and the onset of a briefly presented curved line (the stimulus onset asynchrony, or SOA). For comparison, the "goodness" of the apparent motion is also measured at each SOA (see Kolers \& Touchstone, Note 1). The notion that curvature overshoot is due to eye move- 
ments is also tested. Experiment 2 measures the contribution of classical curvature-contrast effects to curvature overshoot (Gibson, 1933, 1937; Imai, 1956; see Oyama, 1960). Experiment 3 shows that curvature overshoot due to apparent motion cannot be explained as a combination of position overshoots of elementary segments making up the inducing stimuli.

\section{EXPERIMENT 1: APPARENT CURVATURE AS A FUNCTION OF SOA}

The stimulus configuration used to measure curvature overshoot in apparent motion is illustrated in Figure 1 . The spatially fixed straight and curved lines on the left side of the display, separated vertically by a .4-deg visual angle, were each presented briefly for various SOAs. The apparent curvature of the leftside lower line was estimated by being matched with the adjustable curved line on the right side of the display. The "goodness" of any apparent motion was measured by a category rating method. The vertical separation of the left-side straight and curved lines was chosen, on the basis of preliminary measurements, to reduce confounding curvature effects related to the classical curvature-contrast illusions (Imai, 1956; see, also, Gibson, 1933, 1937, and Orbison, 1939). The classical illusion is generated when a curved line is superimposed on a straight line: the straight line appears curved in the direction opposite to the "inducing" curved line. The classical illusion does, however, diminish as the curved and straight lines are spatially separated. As shown in Experiment 2, the

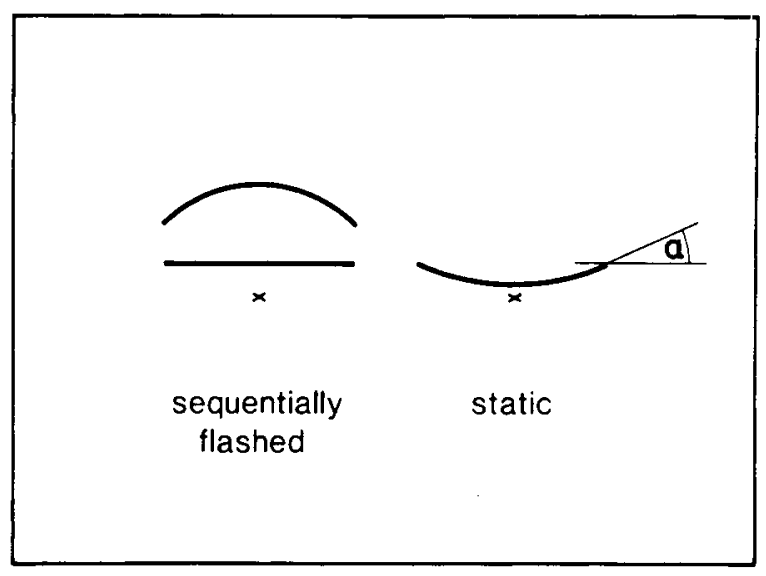

Figure 1. Stimulus display for Experiment 1, shown to scale. The horizontal extent of each of the lines in the display subtended $2.0 \mathrm{deg}$ visual angle. The spatially fixed lines on the left side of the display were each presented for $50 \mathrm{msec}$, at various stimulus onset asynchronies. The variable-curvature line on the right side of the display, used to match the apparent curvature of the left-side straight line, was presented continuously. Curvature was specified by the angle $\alpha$ shown. For the left-side curved line, the magnitude of $\alpha$ was fixed at $\mathbf{4 5 . 0}$ deg. The fixation crosses were used only in a control experiment (see text). present nonoverlapping stimulus configuration is important in permitting a modification by which the classical curvature-contrast effects may be analyzed.

\section{Method}

Stimuli and Apparatus. The stimulus display illustrated in Figure 1 is to scale; the horizontal extent of each of the lines in the display subtended $2.0 \mathrm{deg}$ at the eye. The spatially fixed straight and curved lines on the left side of the display were each presented for $50 \mathrm{msec}$, with various positive and negative SOAs (positive SOAs signifying that the curved line preceded the straight line). The static curve on the right side of the display had smoothly adjustable curvature and was presented continuously during the sequential presentation of the lines on the left side of the display. The fixation crosses were present only in a subsequent control experiment.

Both fixed and adjustable curved lines were arcs of circles, and the curvature of each was specified by the angle $\alpha$ shown in Figure 1. For the left-side curved line, the magnitude of $\alpha$ was $45.0 \mathrm{deg}$.

The stimuli were produced by an on-line computer on the screen of an X-Y display oscilloscope (Hewlett-Packard, Type HP1317A) with P31 phosphor (decay time less than $50 \mu \mathrm{sec}$ ). Each line consisted of 15-17 concatenated line segments and, over the range of curvatures employed here, appeared smooth to the eye. The curvature of the adjustable curved line was controlled by the subject using a rotary potentiometer interfaced to the computer. The spatial fidelity of the system was such that the greatest deviation from specified curve shape was $.2 \mathrm{~min}$ of arc of visual angle. Each complete line on the screen was refreshed at $10-\mathrm{msec}$ intervals. Thus, each of the left-side lines was drawn identically six times to make up the nominal $50-\mathrm{msec}$ duration. This fine temporal structure of the stimuli could not be detected visually, nor did it interfere with the generation of apparent motion.

The display screen was viewed binocularly at a distance of $1.7 \mathrm{~m}$ through a view tunnel and optical system which produced a uniform background field (white, luminance $30 \mathrm{~cd} \mathrm{~m}^{-2}$ ) upon which the stimulus lines appeared superimposed. The intensity of the lines was set by each subject at the beginning of each experimental session so that each line was 80 times increment luminance threshold (typically $50 \mu \mathrm{cd} \mathrm{sec}$ ). (This setting was achieved by introducing a 1.9 log-unit neutral density filter over the stimulus lines and adjusting their intensity to increment threshold on the unattenuated background.)

Procedure. Subjects were instructed to adjust (by means of the rotary control) the curvature of the line on the right side of the display so that it appeared identical to the lower line on the left side of the display. When the match appeared satisfactory, they were to indicate this and the "goodness" of the apparent motion on the left side of the display using one of the following three category ratings: 0 -there was no apparent motion; 1 -some apparent motion was evident but not as marked as in category 2; 2 -one line appeared to move smoothly and continuously into the other (beta motion). The subjects signaled their responses on a pushbutton box interfaced to the computer.

The timing of presentations was as follows. For each fixed SOA, the curved line and straight line were presented in the prescribed order three times at intervals of $2 \mathrm{sec}$. This sequence of presentations could be repeatedly initiated by the subject by pressing a switch on the pushbutton box. The curvature of the right-side line could be altered only in the period between these sequences.

In making a curvature match, the subjects were instructed to alternate fixation from the center of the left-side lower line to the center of the right-side line (Figure 1). Control experiments on the significance of the fixation regime are discussed below.

After a match had been made, and the rating of the apparent motion recorded, a new SOA was selected automatically. The previous setting of the adjustable right-side line was spoiled by the subject before a new match was begun.

Each experimental run consisted of 10 such curvature matches and apparent-motion ratings, with SOA values ranging from 
-200 to $250 \mathrm{msec}$. Each experimental session consisted of a block of five runs, and each subject participated in two sessions. The sequence of SOAs in each run was chosen pseudorandomly but balanced over runs for order and carry-over effects. The subjects were not informed of their performance during the experiment.

Subjects. Five subjects, aged 21 to 36 years, participated in the

$\longrightarrow$ without fixation targets

$\sigma-\infty$ with fixation targets
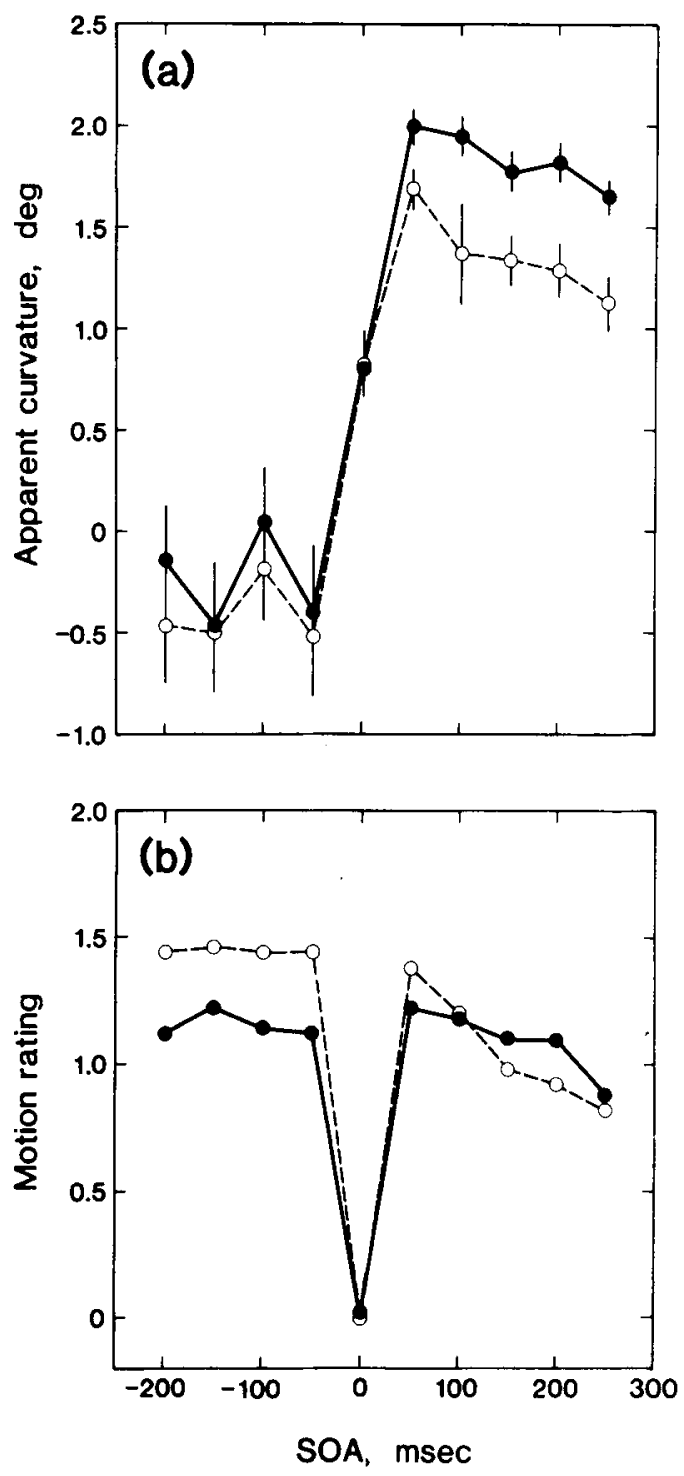

Figure 2. (a) Apparent curvature of the left-side straight line illustrated in Figure 1 as a function of the onset asynchrony between it and the left-side curved line. Positive SOAs signify that the curved line preceded the lower straight line. Curvature is specified by the angle $\alpha$ shown in Figure 1. Data are pooled over five subjects. The vertical bars show \pm 1 SEM. (b) Categorical rating of "goodness" of apparent motion between the two left-side line stimuli illustrated in Figure 1 as a function of SOA. The rating of 2.0 corresponds to beta motion and 0 to no apparent motion. Each point is based on 50 ratings pooled over the five subjects. Points shown by the filled and unfilled circles refer to different fixation regimes used in Experiment 1. experiment; four were male, N.J.L., C.J.H., and the two authors, S.G. and D.H.F., and one was female, R.S.S. Each had normal or corrected-to-normal vision (Snellen acuities each not worse than 6/6). R.S.S., N.J.L., and C.J.H. were unaware of the purpose of the experiment.

\section{Results}

Individual subjects' results were similar, and in Figure 2 the filled circles joined by the continuous lines show the pooled data. In Figure $2 a$ the apparent curvature of the left-side lower line (see Figure 1), estimated by the matching procedure described in the above Method section, is plotted against SOA, positive SOAs signifying that the left-side curved line preceded the left-side straight line. Curvature is expressed in terms of the angle $\alpha$ indicated in Figure 1. (Recall that the magnitude of $\alpha$ for the left-side curved line was fixed at 45.0 deg.) Each point is based on 50 curvature matches, and the vertical bars indicate \pm 1 SEM. In Figure 2b, the mean rating of the "goodness" of the apparent motion is plotted against SOA. Each point is based on 50 ratings, where the maximum rating of 2.0 corresponds to beta motion, and the minimum rating of 0 to no apparent motion. (Data shown by the unfilled circles connected by the broken lines refer to a control experiment described later.)

From Figure $2 a$, it is evident that apparent curvature was a strongly asymmetric function of SOA: there was marked apparent curvature at positive SOAs and little apparent curvature at negative SOAs. Maximum apparent curvature occurred at $50 \mathrm{msec}$ SOA, although its subsequent decline with increasing SOA was small. In contrast, the "goodness" of the apparent motion produced by the stimuli (Figure $2 b$ ) was approximately symmetric about zero SOA.

At zero SOA, at which no apparent motion was reported (Figure $2 \mathrm{~b}$ ), there was highly significant nonzero apparent curvature (Figure 2a) $[\mathrm{t}(49)=5.72$, $\mathrm{p}<.001]$, but its magnitude was very significantly lower than all values at positive SOAs $[t(98) \geqslant 5.16$, $\mathrm{p}<.001$ in all cases]. Apparent curvature at negative SOAs was not significantly different from zero $[\mathrm{F}(4,196)=1.11, \mathrm{p}>.2]$.

\section{Discussion}

Magnitude of curvature overshoot. In assessing the magnitude of the measured apparent curvature, it is useful to compare it with the threshold for the detection of curvature in a straight line. For curved lines of the lengths used here, acuity for curvature corresponds to values of the angle $\alpha$ (Figure 1) of the order of .3 deg (Andrews, Butcher, \& Buckley, 1973). Maximum apparent curvature (Figure 2a) was more than six times this acuity limit.

The fact that the apparent curvature of the straight line at zero SOA (at which there was no apparent motion, Figure 2b) was significantly lower than the apparent curvature of the straight line at positive SOAs 
(at which there was apparent motion) suggests that curvature overshoot is not a simple consequence of classical curvature-contrast effects (see, particularly, Imai, 1956). Indeed, separate measurement of the apparent-curvature effect induced when the curved line and straight line were presented simultaneously for several seconds gave values not greater than that shown at zero SOA in Figure $2 a$.

A more complex hypothesis might be contrived, however, in which it was supposed that curvaturecontrast processes underlie not only the apparentcurvature effect at zero SOA, but, by some unspecified spatiotemporal facilitation, also the greater effects at positive SOAs. The existence of apparent motion at positive SOAs might then be regarded as merely coincidental to the curvature-overshoot phenomenon. In fact, as we show in Experiment 2, there is a highly significant curvature overshoot attributable solely to apparent motion.

Visual tracking. An alternative explanation of the results of Figure 2a, still related to the classical curvature effects of Imai (1956) and Gibson (1933, 1937), might be constructed as follows. Suppose that, instead of alternating fixation from the center of the left-side lower line to the center of the right-side line (Figure 1), subjects inadvertently visually tracked the illusory figure undergoing apparent motion. Then, at the end of the motion, the curved line and straight line would have occupied the same region of the retina and might thus have generated a strong curvaturecontrast effect. It would be necessary, of course, to suppose a temporal asymmetry in this hypothesized process to account for the asymmetric effects demonstrated when the presentation order was reversed (Figure 2a).

To test this tracking hypothesis, two control experiments were performed. In the first, the experiment described above was repeated but a change made in the fixation conditions with the introduction of two steady fixation targets, as shown in Figure 1. The targets consisted of two crosses placed $20 \mathrm{~min}$ of arc below the centers of the left-side lower and right-side lines. The subjects were instructed to alternate fixation from one cross to the other during the curvature-matching task. (The subjects reported that, under this regime, accurate fixation could be easily maintained.) The results of this experiment are shown in Figure 2 by the unfilled circles joined by the broken lines. The form of the dependence of apparent curvature and of motion rating on SOA is evidently similar to that for the previous experiment without steady fixation targets (filled circles). Apart from a small downward shift in apparent curvature (recall that the stimuli had different retinal locations here), differences between the results for this and for the previous fixation condition were not significant $[F(10,490)=.47, p>.5)$. There was also no significant change in motion rating $(\mathrm{z}=.94, \mathrm{p}>.2) .^{1}$
The second control experiment was based on the original informal demonstration of curvature overshoot involving the sequential presentation of a circle and an overlapping square, as described in the introduction. There the final form of the illusory apparentmotion figure appeared curved inwards on all four sides. Since the four components of the inward-going apparent motion (top, bottom, left, and right) could not be tracked simultaneously, the phenomenon, in that case, could not be attributed to inappropriate patterns of eye fixations.

The purpose of the second control experiment was to provide confirmation of this result for the nonoverlapping stimuli used in the present experiment. The whole left side of the stimulus display illustrated in Figure 1 was mirror-imaged in the region below the left-side lower line in Figure 1. Thus, .4 deg below the lower left-side line, there appeared a second straight line, and below that, at the appropriate distance, an inverted copy of the left-side curved line. The two oppositely curved lines were presented simultaneously for $50 \mathrm{msec}$, as were the two straight lines. For a positive SOA of $50 \mathrm{msec}$, at which strong apparent motion was obtained before (Figure $2 \mathrm{~b}$ ), all subjects reported that the two curved lines appeared to change simultaneously into two lines with opposite curvatures in the directions dictated by the two stimulus sequences. The notion that the apparent curvature shown in Figure $2 a$ is a consequence of visual tracking was rejected.

\section{EXPERIMENT 2: APPARENT CURVATURE DUE TO APPARENT MOTION}

It was suggested in the Discussion of Experiment 1 that apparent motion from the curved line to the straight line (Figure 1) need not itself have been the cause of the apparent curvature of the straight line. In this experiment, we tested that hypothesis by repeating the main experiment of the previous section, but under conditions in which (1) the apparent motion that occurred there at positive (and negative) SOAs (Figure $2 \mathrm{~b}$ ) was largely suppressed, and (2) the operation of any curvature-contrast effects was unimpaired.

To achieve this selective suppression of apparent motion between the curved line and straight line, we introduced a second horizontal straight line into the left side of the display so that it was tangential to the curved line, as in Figure 3. This second straight line was presented in synchrony with the lower straight line. It was reasoned that, at nonzero SOAs, apparent motion would be most likely to occur between the curved line and the closest of the two straight lines, that is, the upper straight line (an assumption supported by exploratory measurements and confirmed more formally below). Because the stimulus 


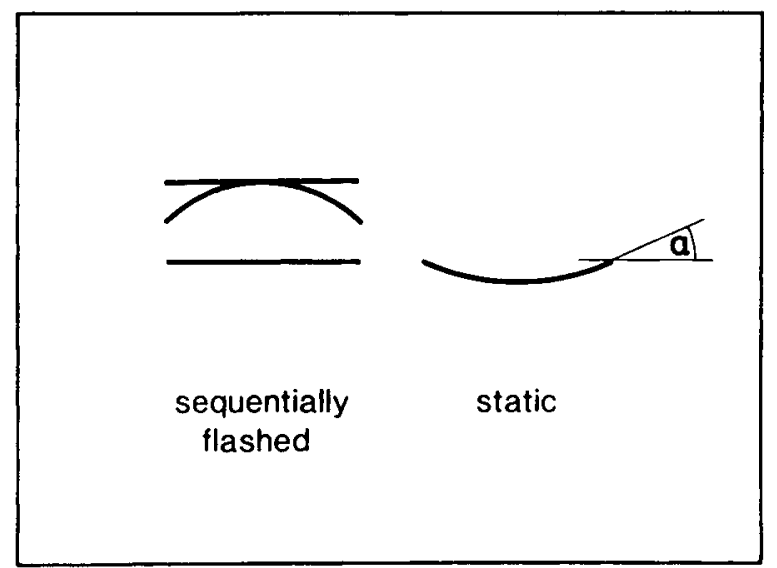

Figure 3. Stimulus display for Experiment 2, shown to scale. The configuration was identical to that used in Experiment 1 (Figure 1), except that the upper straight line on the left side of the display, tangential to the curved line, could be presented in synchrony with the lower straight line on the left of the display.

field below the curved line was precisely the same as in Experiment 1, it was reasoned that curvaturecontrast effects, for zero or positive SOAs, should be the same. (Any influence of the upper straight line on the curvature-contrast-inducing effect of the curved line was expected to be negligible, as also confirmed below.)

The experiment was performed in an interleaved fashion: runs in which the upper straight line was presented were alternated with runs in which it was absent. The latter runs provided a matched control by repeating Experiment 1 . If the hypothesis that curvature-contrast effects underlie the curvature overshoot shown in Figure $2 a$ were correct, then the results of these interleaved runs should be the same.

\section{Method}

Stimuli and Apparatus. The arrangement of the stimuli illustrated in Figure 3 is to scale, and, apart from the introduction of the upper straight line on the left side of the display, the configuration was identical to that used in Experiment 1 . On the left side, the upper straight line appeared at the same time and for the same duration, $50 \mathrm{msec}$, as the lower straight line; the curved line had a duration of $50 \mathrm{msec}$ also. The time courses of the stimuli and the range of SOAs were identical to those used in Experiment 1 .

Procedure. Subjects were informed that there were two types of run: those in which the upper straight line was presented, with time course as described above, and those in which it was absent. For both types of run, they were instructed to adjust the curvature of the right-side adjustable curved line (by means of the rotary control; see Method section, Experiment 1) so that it appeared identical with the bottom line on the left side of the display. When the match appeared satisfactory, they were to indicate this and the "goodness" of the apparent motion between the left-side curved line and bottom line, using the category ratings $0,1,2$, as defined in the Method section of Experiment 1. It was stressed that they were to ignore any apparent motion between the curved line and the top line on those trials in which the latter was presented.

The cycling of the presentations, fixation regime, and ordering of SOAs within each run were all as in the main part of Experi- ment 1. Runs with and without the upper straight line were alternated, with the same sequence of SOAs used in each pair.

Subjects. The subjects of Experiment 1 participated in this experiment. R.S.S., N.J.L., and C.J.H. were unaware of the purpose of the experiment.
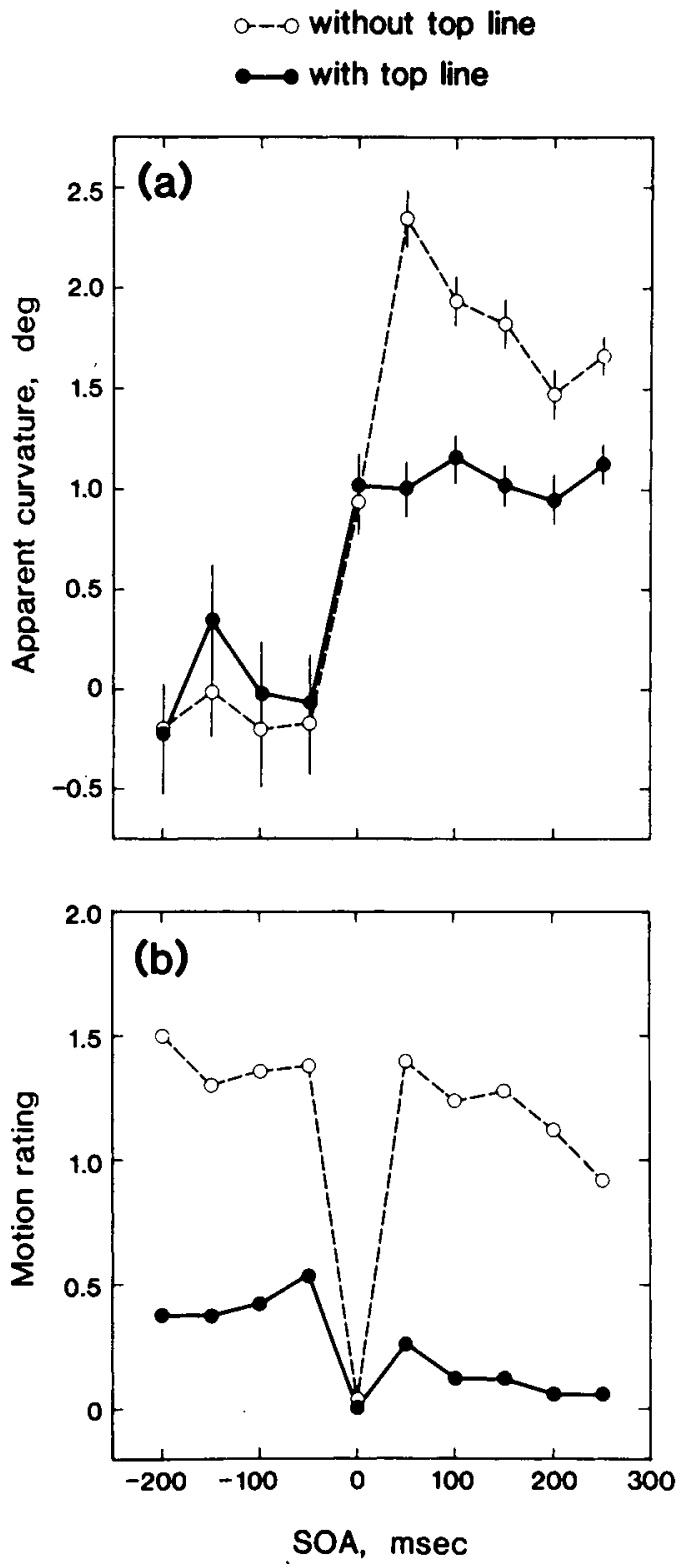

Figure 4. (a) Apparent curvature of the left-side lower straight line in Figure 3 as a function of onset asynchrony between it and the left-side curved line. Positive SOAs signify that the curved line preceded the straight line. Data are pooled over five subjects. The vertical bars show \pm 1 SEM. (b) Categorical rating of "goodness" of apparent motion between the left-side curved and lower straight lines (Figure 3) as a function of SOA. The rating of 2.0 corresponds to beta motion and 0 to no apparent motion. Each point is based on 50 ratings pooled over the five subjects. Points shown by the filled circles were obtained in runs in which the left-side upper straight line (Figure 3) was presented; points shown by the unfilled circles were obtained in runs in which the left-side upper straight line was absent. 


\section{Results}

Individual subjects' results were similar, and Figure 4 shows the pooled data. In Figure $4 a$, the apparent curvature of the left-side lower straight line (Figure 3) is plotted against SOA, where positive SOAs signify, as before, that the curved line preceded the lower straight line. Curvature is expressed in terms of the angle $\alpha$ (Figure 3). Each point in Figure $4 \mathrm{a}$ is based on 50 curvature matches, and the vertical bars indicate \pm 1 SEM. In Figure $4 \mathrm{~b}$, the mean rating of the "goodness" of the apparent motion between the left-side curved line and lower straight line is plotted against SOA. Each point is based on 50 ratings, where 2.0 corresponds to beta motion and 0 to no apparent motion. In both Figures $4 \mathrm{a}$ and $4 \mathrm{~b}$, points indicated by the filled circles and joined by the continuous lines were obtained in runs in which the upper straight line (Figure 3) was presented; those points indicated by the unfilled circles and joined by the broken lines were obtained in runs in which the upper straight line was absent.

Those runs without the upper straight line replicated Experiment 1, and the results (unfilled circles in Figures $4 a$ and $4 b$ ) were not significantly different from the results shown in Figures $2 \mathrm{a}$ and $2 \mathrm{~b}$ (filled circles) [respectively, $F(10,490)=0.72, p>.5$, and $z=1.23$, $\mathrm{p}>.2] .{ }^{1}$ The peak in apparent curvature at $50 \mathrm{msec}$ SOA is, however, particularly evident here. For those runs in which the upper straight line was presented (results shown by the filled circles in Figures $4 \mathrm{a}$ and $4 \mathrm{~b}$ ), apparent curvature at negative and zero SOAs was not significantly different from that for runs in which the upper straight line was absent $[F(5,245)=.29, p>.5]$, but was highly significantly different at positive $\operatorname{SOAs}[\mathrm{F}(5,245)=24.93$, $\mathrm{p}<.001]$. In addition, the "goodness" of the apparent motion between the curved line and the lower straight line was markedly diminished over all SOAs; mean rating over all SOAs was reduced to .20 of the same rating when the upper straight line was absent (a highly significant reduction, $z=6.07, p<.001)^{1}$

\section{Discussion}

Apparent-motion curvature overshoot. The immediate object of this experiment was to test whether apparent curvature of the left-side lower straight line (Figure 3) would be the same with and without presentation of the upper straight line. The results of Figure 4a show clearly that apparent curvature was sensitive to the presentation of the upper straight line. How do these results relate to apparent-motion and curvature-contrast effects? When the upper straight line was presented, apparent motion was certainly greatly diminished (Figure $4 \mathrm{~b}$, filled circles), whereas apparent curvature at zero SOA (Figure 4a, filled circles), previously attributed to curvature- contrast effects (see Discussion, Experiment 1), was not significantly altered $[\mathrm{t}(98)=.37, \mathrm{p}>.2]$. The rationale offered earlier concerning the action of the upper straight line on the production of apparent motion and apparent curvature would seem justified, and the data shown in Figure 4a by the filled circles should accordingly represent curvature-contrast effects alone. Apparent curvature of the lower straight line due to such effects thus appears to be zero at negative SOAs, when the curved line followed the lower straight line, and nonzero and constant at nonnegative SOAs, when the curved line preceded or occurred at the same time as the straight line (at least over the present range of SOAs). From the above argument, it follows that the data shown in Figure 4a by the unfilled circles represent a varying combination of apparent-motion and curvature-contrast effects. It is evident that, at positive SOAs, there is a substantial contribution to apparent curvature from apparent-motion effects alone.

Although apparent curvature due to curvature contrast and to apparent motion need not be simply additive, it is instructive to take the differences of the sets of data in Figure $4 a$ and of the sets in Figure $4 b$ to obtain an indication of the apparent-curvature dependence arising solely from apparent motion. Figures $5 \mathrm{a}$ and $5 \mathrm{~b}$ show the results. In Figure 5a, net apparent curvature is not significantly different from zero over negative and zero SOAs $[F(5,245)=.66, p>.5]$; it is highly significantly nonzero at all positive SOAs $[F(5,245)=24.34, p<.001]$. The "goodness" of netapparent motion (Figure 5 b) is relatively high at both positive and negative SOAs, and zero at zero SOA.

To summarize the implications of the present experiment: in the display of Figure 1, the left-side lower straight line underwent an apparent curvature due solely to apparent motion between it and the curved line, and this apparent curvature occurred only when the curved line preceded the straight line, that is, when the straight line was the "target form" for the illusory figure undergoing the apparent motion.

Decomposition hypothesis. Given that there is an apparent-motion curvature overshoot, can it be explained by decomposition into many elementary apparent-motion position overshoots between pairs of vertically aligned points on the curved and straight lines? Those points on the curved line near its ends (Figure 1) have less vertical distance to travel in apparent motion than do those points near its center; if the amount of position overshoot of these points, now regarded as undergoing linear apparent motion, increased with the vertical distance to be covered, then the points near the center of the curved line should overshoot more than those near the ends. The combined effect of these elementary position overshoots could result in an illusory curvature of the 

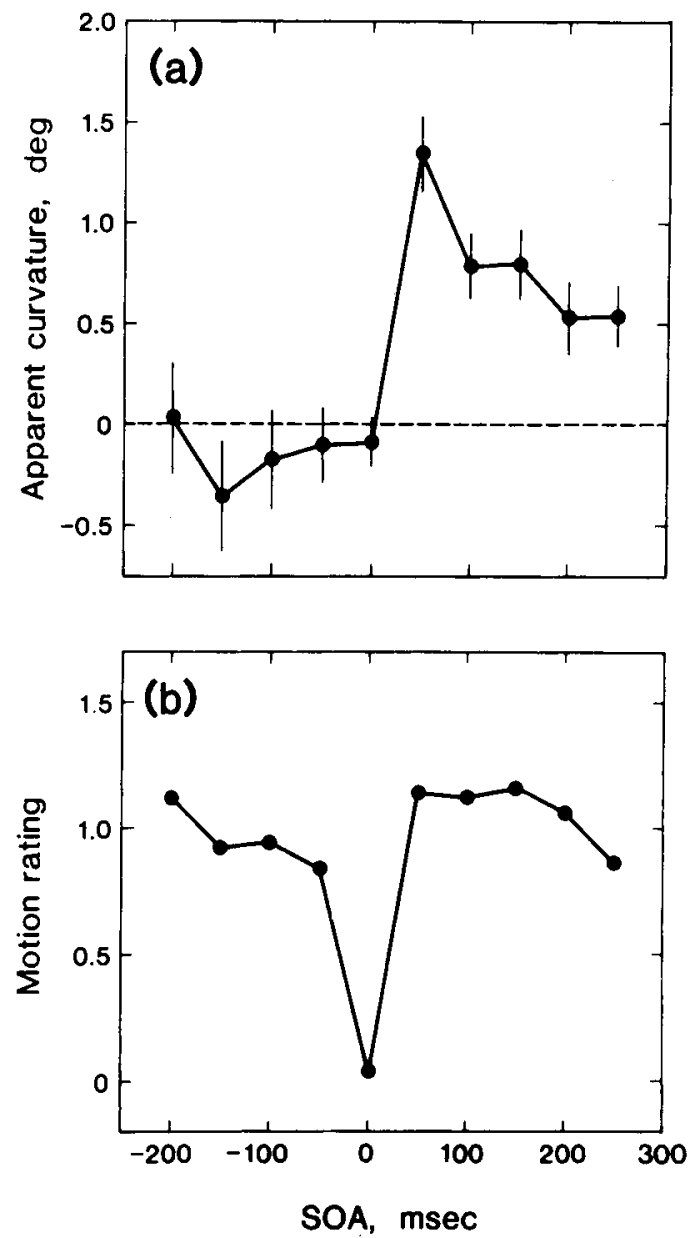

Figure 5. (a) Difference in apparent curvatures of the left-side lower straight line in Figure 3, without and with presentation of the upper straight line, as a function of onset asynchrony between the lower straight line and the left-side curved line. Positive SOAs signify that the curved line preceded the straight line. The vertical bars show \pm 1 SEM. (b) Difference in categorical rating of "goodness" of apparent motion between the left-side curved and lower straight lines (Figure 3) without and with presentation of the upper straight line as a function of SOA. The rating of 2.0 corresponds to beta motion and 0 to no apparent motion. Data in (a) and (b) are means of matched differences taken from the raw data on which Figure 4 was based.

left-side lower line (Figure 1) in the direction observed. This hypothesis was tested in the following experiment.

\section{EXPERIMENT 3: POSITION OVERSHOOT AS A FUNCTION OF SPATIAL SEPARATION}

In this experiment, position overshoot was measured for the sequential presentation of two small line segments, as illustrated on the left side of Figure 6. Their SOA was fixed at the value found for maximum curvature overshoot (and "goodness" of apparent motion) in Experiments 1 and 2, namely $50 \mathrm{msec}$ (the apparent motion being downward). For each vertical separation of the two left-side segments, the apparent vertical position of the left-side lower segment was estimated by matching it against the vertically adjustable right-side segment. The separation of the two left-side segments (only the top segment being displaced) was varied over the range of separations defined by vertically aligned pairs of points on the curved and straight lines of Figure 1.

To successfully explain curvature overshoot by the decomposition hypothesis proposed at the end of the previous section, it is necessary that, in the present experiment, any apparent downward displacement of the lower segment should increase systematically with the increasing vertical separation of the left-side segments. The magnitude of this required increase is given below.

\section{Method}

Stimuli and Apparatus. The full stimulus display is illustrated, to scale, in Figure 6. Each line segment subtended .125 deg at the eye. The two line segments on the left side of the display were each presented for $50 \mathrm{msec}$, with SOA fixed at $50 \mathrm{msec}$, the upper segment preceding the lower segment. The vertical separation of the two left-side segments was varied from .4 to $.8 \mathrm{deg}$ (the position of the lower segment remaining fixed throughout). The vertical position, $y$, of the line segment on the right side of the display could be varied continuously. Other details were as for Experiment 1 .

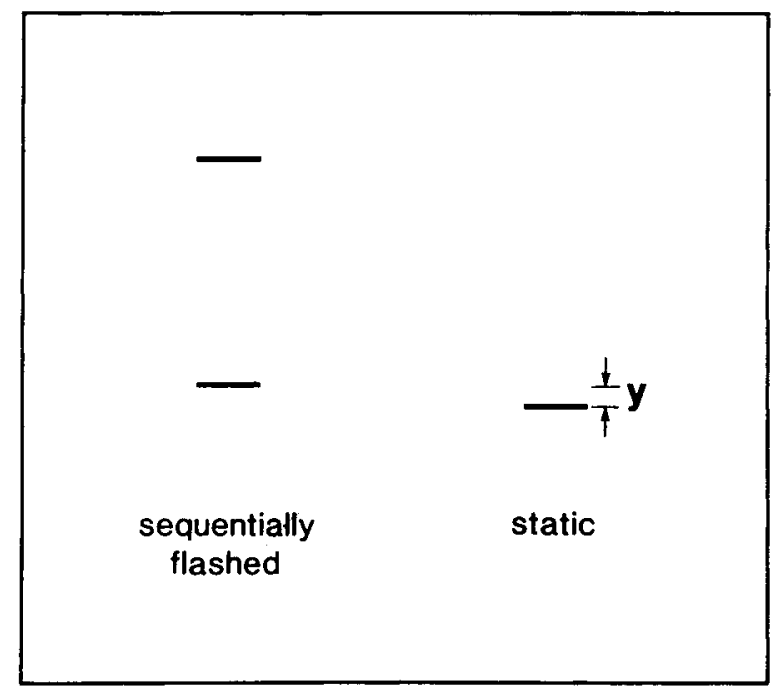

Figure 6. Stimulus display for Experiment 3, shown to scale. The horizontal extent of exch of the line segments in the display subtended $\mathbf{. 1 2 5}$ deg visual angle. The two line segments on the left side of the display were each presented for $50 \mathrm{msec}$, with stimulus onset asynchrony fixed at $\mathbf{5 0}$ msec, the lower segment following the upper segment. The position of the left-side lower segment was fixed, and the position of the left-side upper segment was varied vertically so that it was between .4 and $.8 \mathrm{deg}$ above the lower segment. The vertically adjustable line segment on the right side of the display, used to match the apparent vertical position of the leftside lower segment, was presented continuously. Apparent position was specified by the coordinate $y$ shown. 
Procedure. Subjects were instructed to adjust (by means of the rotary control) the vertical position of the right-side segment (Figure 6) so that it appeared to be vertically in line with the leftside lower segment. After a position match had been made, subjects reported on the "goodness" of the apparent motion using the category rating method described in Experiment 1. The cycling of stimulus presentations and the fixation regime were also as in the main part of Experiment 1.

Each experimental run consisted of six position matches, each with a different vertical separation of the left-side segments. Each subject participated in one experimental session comprising six runs. The sequence of vertical separations of the left-side segments used in each run was chosen pseudorandomly, but balanced over runs for order and carry-over effects. Subjects received no information concerning their performance.

Subjects. The subjects of Experiment 1 participated in this experiment. R.S.S., N.J.L., and C.J.H. were unaware of the purpose of the experiment.

\section{Results}

The results were similar for the five subjects, and Figure 7 shows the pooled data. In Figure $7 \mathrm{a}$, the apparent vertical position (downward positive) of the left-side lower line segment (Figure 6), expressed in minutes of arc visual angle relative to its true position, is plotted as a function of the vertical separation of the left-side line segments, also in minutes of arc. Each point is based on 30 position matches, and the vertical bars show \pm 1 SEM. In Figure $7 b$, the mean "goodness" of the apparent motion is shown against the corresponding vertical separation of the segments. Each point is the mean of 30 ratings, where 2.0 corresponds to beta motion and 0 to no apparent motion (see Method section, Experiment 1).

There was an evident overall positive positional overshoot (Figure 7a) of about 1.6 min of arc, but, critically for the decomposition hypothesis under test, there was little systematic increase in the magnitude of position overshoot with increase in segment separation. The solid straight line in Figure $7 a$ is a least squares regression fit to the position-overshoot data. There is no significant nonlinear trend $[F(4,174)=$ $.72, \mathrm{p}>.5]$, and the linear trend is not significantly different from zero $[F(1,174)=.95, p>.2]$. The gradient of the regression line is .0093 (SEM .0079), whereas the gradient required by the decomposition hypothesis is .0289 , three times the observed value. The broken line in Figure 7a shows the line required by the hypothesis, with vertical position adjusted for best fit to the data; it is significantly different from the actual regression line $(\mathrm{z}=2.47, \mathrm{p}<.02)$ and significantly different from the original data points $[F(1,174)=4.22, p<.05]$. Note, incidentally, that the rating of the apparent motion (Figure $7 \mathrm{~b}$ ) was substantially independent of segment separation (compare variation in apparent-motion rating with SOA in Figure $2 b$ ).

\section{Discussion}

The main result of this experiment is that there is no significant increase in position overshoot of a line
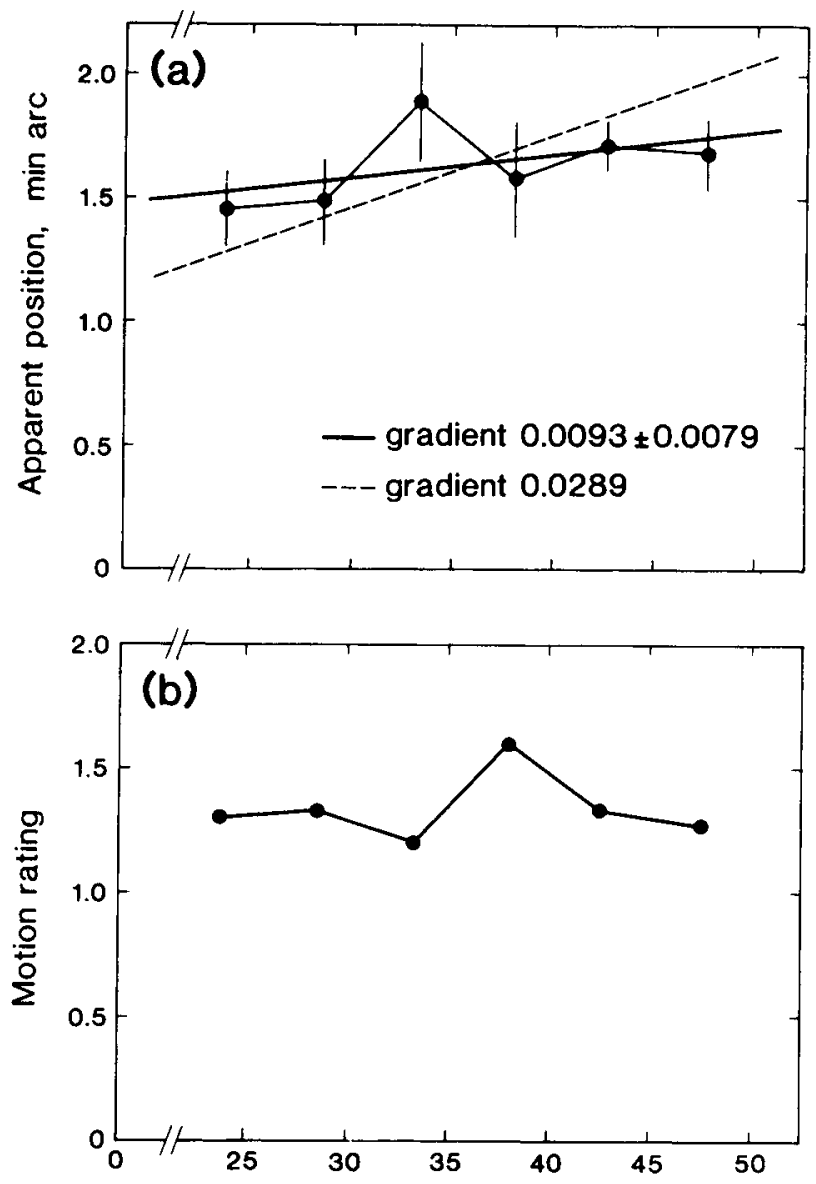

Segment separation, $\min$ arc

Figure 7. (a) Apparent position of the left-side lower line segment illustrated in Figure 6 as a function of the separation of the left-side upper and lower segments. Apparent position (position overshoot) is specified by the coordinate y shown in Figure 6 . Stimulus onset asynchrony is fixed at 50 msec. Data are pooled over five subjects. The vertical bars show \pm 1 SEM. The solid straight line is a least squares regression fit to the data; the broken line is a least squares fit of the hypothetical line describing position overshoot as a function of segment separation according to the decomposition hypothesis (see text). (b) Categorical rating of "goodness" of apparent motion between the two left-side segment stimuli illustrated in Figure 6 as a function of segment separation. The rating of 2.0 corresponds to beta motion and 0 to no apparent motion. Each point is based on 30 ratings pooled over the five subjects.

segment with increasing separation of the segments inducing apparent motion, at least over the range of segment separations of concern here. The hypothesis that the apparent-motion curvature-overshoot results in Experiment 2 may be explained by a decomposition into position overshoots appears untenable.

It should perhaps be noted that the present data do not apply immediately to the question of whether there is an increase in the apparent separation of two lines inducing beta motion. Only the apparent vertical position of the lower, terminal line was measured 
here-no measurements were made of the apparent vertical position of the upper, initial line (compare Caelli, Hoffman, \& Lindman, 1978, Neuhaus, 1930, Scholz, 1924, and Kolers \& Touchstone, Note 1).

Position overshoot in classical apparent motion should also be distinguished from a position overshoot associated with the "fine-grain movement illusion" (Thorson, Lange, \& Biederman-Thorson, 1969; Biederman-Thorson, Thorson, \& Lange, 1971; Foster, 1977). The latter illusion is elicited by the sequential stimulation of two spatially unresolved points in the peripheral field of vision; the extent of the illusion induced by two such points separated by say $.2 \mathrm{deg}$ visual angle may range from 2 to $6 \mathrm{deg}$ as stimulus eccentricity increases from 10 to $24 \mathrm{deg}$ (Foster, Thorson, Mcllwain, \& Biederman-Thorson, 1981).

\section{GENERAL DISCUSSION}

\section{Summary}

When apparent motion occurs between a suitably presented curved line and straight line, the illusory object moving and changing from the first to the second form overshoots its target form and gains a small curvature in the opposite sense. This curvature overshoot appears not to be attributable solely to classical curvature-contrast effects, or to inappropriate patterns of eye fixations. Moreover, it seems not to be explainable in terms of a combination of elementary position overshoots which occur with some forms of linear apparent motion.

\section{Two Schemes for Apparent Motion}

In attempting to explain qualitatively apparentmotion curvature overshoot in relation to current theories of visual apparent motion, we have considered two schemes that might provide possible starting points. The first scheme is based on Caelli and Dodwell's (1980) network model for apparent motion. A distribution of nervous activity is assumed to be generated according to an iterative process which entails a series of weighted summations that build up into a smooth continuous motion. Computations from the model by Caelli and Dodwell (1980) correctly predict an enhancement of the Poggendorf illusion under apparent-motion conditions (Caelli \& Dodwell, 1980). We have not made analogous computations for the present stimuli, but a priori such a scheme would seem to be a plausible candidate for an interpretation of curvature overshoot in terms of neural-net activity.

The second explanatory scheme, which is more cognitive in character, is based on a dynamical description of apparent motion proposed by Foster $(1975,1978)$. It is supposed that beta motion is modeled internally according to approximate analogs of the laws that govern the motion of objects in physical three-dimensional space. (Farrell \& Shepard, 1981, and Shepard, 1980, have developed similar ideas; see also Cooper \& Shepard, 1978, and Shepard \& Judd, 1976.) Thus, beta motion is represented as a trajectory within some internal space of geometrical transformations according to a principle of "least energy" or "least action." This scheme correctly predicts the nonlinear and three-dimensional paths followed by some illusory transforming objects undergoing beta motion. Within such a scheme, a mass-like attribute may be naturally attached to the motion in this space of transformations in such a way that a "ballistic" overshoot of the end point of the trajectory then follows. The form of the overshoot would depend on the preceding part of the trajectory: for linear beta motion, the extrapolation would constitute a position overshoot; for beta motion involving changes in curvature, the extrapolation would constitute a curvature overshoot.

\section{REFERENCE NOTE}

1. Kolers, P. A., \& Touchstone, G. E. Variations of perceived distance with apparent motion (Quarterly Progress Report No. 79). Cambridge: Research Laboratory of Electronics, Massachusetts Institute of Technology, 1965.

\section{REFERENCES}

Andrews, D. P., Butcher, A. K., \& Buckley, B. R. Acuities for spatial arrangement in line figures: Human and ideal observers compared. Vision Research, 1973, 13, 599-620.

Biederman-Thorson, M., Thorson, J., \& Lange, G. D. Apparent movement due to closely spaced sequentially flashed dots in the human peripheral field of vision. Vision Research, 1971, 11, 889-903.

Caelli, T. M., \& Dodwell, P. C. On the contours of apparent motion: A new perspective on visual space-time. Biological Cybernetics, 1980, 39, 27-35.

Caelli, T. M., Hoffman, W. C., \& Lindman, H. Apparent motion: Self-excited oscillations induced by retarded neuronal flows. In E. L. J. Leeuwenberg \& H. F. J. M. Buffart (Eds.), Formal theories of visual perception. Chichester: Wiley, 1978.

Cooper, L. A., \& Shepard, R. N. Transformations on representations of objects in space. In E. C. Carterette \& M. P. Friedman (Eds.), Handbook of perception (Vol. 8). New York: Academic Press, 1978.

ExNER, S. Experimentelle Untersuchung der einfachsten psychischen Processe. Pffügers Archiv für die gesamte Physiologie, $1875,11,403-432$.

Farreld, J. E., \& Shepard, R. N. Shape, orientation, and apparent rotational motion. Journal of Experimental Psychology: Human Perception and Performance, 1981, 7, 477-486.

Foste R, D. H. Visual apparent motion and some preferred paths in the rotation group $S O(3)$. Biological Cybernetics, 1975, 18, 81-89.

Foste R, D. H. Rod- and cone-mediated interactions in the finegrain movement illusion. Vision Research, 1977, 17, 123-127.

Foster, D. H. Visual apparent motion and the calculus of variations. In E. L. J. Leeuwenberg \& H. F. J. M. Buffart (Eds.), Formal theories of visual perception. Chichester: Wiley, 1978.

Foster, D. H., Thorson, J., Mcllwain, J. T., \& BiedermanThorson, M. The fine grain movement illusion: A perceptual probe of neuronal connectivity in the human visual system. Vision Research, 1981, 21, 1123-1128. 
Gibson, J. J. Adaptation, after-effect, and contrast in the perception of curved lines. Journal of Experimental Psychology, $1933,16,1-31$.

Gibson, J. J. Adaptation with negative after-effect. Psychological Review, 1937, 44, 222-244.

ImAI, S. Illusions in the figure consisting of straight and curved lines. Japanese Journal of Psychology, 1956, 27, 147-149, 235-237.

KENKEL, F. Untersuchungen über den Zusammenhang zwischen Erscheinungsgrösse und Erscheinungsbewegung bei einigen sogenannten optischen Täuschungen. Zeitschrift für Psychologie, $1913,67,358-449$.

Kolers, P. A. Aspects of motion perception. Oxford: Pergamon, 1972.

Kolers, P. A., \& Pomerantz, J. R. Figural change in apparent motion. Journal of Experimental Psychology, 1971, 87, 99-108.

Neuhaus, W. Experimentelle Untersuchung der Scheinbewegung. Archiv für die gesamte Psychologie, 1930, 75, 315-458.

Orbison, W. D. Shape as a function of the vector-field. American Journal of Psychology, 1939, 52, 31-45, 309.

Oyama, T. Japanese studies on the so-called geometrical-optical illusions. Psychologia, 1960, 3, 7-20.

Scноцz, W. Experimentelle Untersuchungen über die phänomenale Grösse von Raumstrecken, die durch Sukzessiv-Darbietung zweier Reize begrenzt werden. Psychologische Forschung, 1924, 5, 219-272.

ShePard, R. N. Psychophysical complementarity. In M. Kubovy \& J. R. Pomerantz (Eds.), Perceptual organization. Hillsdale, N.J: Erlbaum, 1981.
Shepard, R. N., \& JudD, S. A. Perceptual illusion of rotation of three-dimensional objects. Science, 1976, 191, 952-954.

Thorson, J., Lange, G. D., \& Biederman-Thorson, M. Objective measure of the dynamics of a visual movement illusion. Science, 1969, 164, 1087-1088.

Wertheimer, M. Experimentelle Studien über das Sehen von Bewegung. Zeitschrift für Psychologie, 1912, 61, 161-265. [Translated in part in T. Shipley (Ed.), Classics in psychology. New York: Philosophical Library, 1961.]

\section{NOTE}

1. Motion ratings to be compared were paired on the basis of SOA and a standard regression analysis performed. Under the hypothesis that the two sets of ratings have the same underlying distribution, the gradient, $g$, of the regression line should be unity. This was tested by computing the SEM, $s$, of $g$ and comparing the quantity $(1-\mathrm{g}) / \mathrm{s}$ with the standard normal variable. For the analysis of the pairs of motion ratings in the text, the gradient was 1.17 in Experiment 1 (Discussion, Figure 2b, filled and unfilled circles), 1.13 in the first comparison in Experiment 2 (Results, Figure 4b, unfilled circles, and Figure $2 \mathrm{~b}$, filled circies), and .29 in the second comparison in Experiment 2 (Results, Figure 4b, unfilled and filled circles). In this last comparison, the two sets of ratings were, as expected, substantially different.

(Manuscript received June 22, 1981; revision accepted for publication January 4,1982 .) 